The cardiac depressant effect of beta-blockers is widely quoted, but exactly what is meant by the term needs to be specified. The ailing heart has two compensatory mechanisms, both of which can be interfered with by beta-blockade. By the intrinsic Frank-Starling mechanism the increased diastolic stretch inevitable after an increase in venous return brings about an increased force of contraction and higher stroke volume. Secondly, there is a humoral action through which sympathetic stimulation increases the heart rate when the stroke volume is low and maintains contractile strength at maximum. The performance of the heart may decline after slowing of its rate, a fall in venous return, interference with atrioventricular conduction, or a decrease in the force of contraction, any one of which may be the means whereby the beta-blocker causes its adverse effect in a particular patient. Conversely these same actions can in other circumstances improve function, as in angina by reducing metabolic demand; in mitral stenosis by giving time for the left atrium to empty; and in dysrhythmia through slowing conduction time.

Other side effects of beta-blockers are less serious and usually dose related. There has been recent interest in their psychotropic effects ${ }^{5}$ and particularly in an hallucinogenic action in a few patients. Drowsiness, lassitude, and depression may occur, particularly with prolonged high dose treatment. Special caution is needed in the elderly, in those with poor renal function, and in those with cardiac failure or recent acute myocardial infarction. ${ }^{6}$ Reduction of the sympathetically stimulated contractile force of the muscle is the least important of all the beta-blocking effects, but patients with advanced myocardial failure should be fully digitalized-remembering that beta-blockers have a synergistic effect with digitalis in slowing the heart rate in atrial fibrillation. Unlike digoxin beta-blockers can be used to slow the heart in sinus rhythm. Diuretics can be used to counteract any sodium retaining effect of the beta-blocking drugs.

While it is important to stress the potential hazards of these drugs it is also important to stress their safety in patients with uncomplicated angina or hypertension. In angina the benefit is directly attributable to reduction in the work of the heart. Since, however, the onset of pain is preceded by depression of myocardial function from hypoxia, and disappearance of pain is associated with recovery of function, the prevention of angina by beta-blockers is not accompanied by a risk of provoking heart failure-except in patients who have already suffered serious ventricular damage.

There seems little to choose between the three drugs in the incidence of adverse reactions or between propranolol and oxprenolol in the treatment of angina or hypertension. Practolol has been shown to be less effective in the relief of angina ${ }^{8}$; partly because it is less successful in reducing the heart rate during exercise, but also because its lack of peripheral action results in a less marked fall in cardiac output, and thus of ventricular size and work, than occurs with propranolol and oxprenolol. For the same reason practolol is to be preferred in the treatment of dysrhythmias, particularly in patients with a compromised myocardium as in congestive cardiomyopathy or after acute myocardial infarction and it can be tried with caution in patients with obstructive airways disease who have angina.

1 Black, J. W., Crowther, A. F., Shanks, R. G., Smith, L. H., and Dornhorst, A. C., Lancet, 1964, 1, 1080.

2 Brunner, H., Hedwall, P. R., Maier, R., and Meier, M., Postgraduate Medical fournal, 1970, suppl., 46, 5 .

Barrett, A. M., Postgraduate Medical fournal, 1971, suppl., 47, 7. Greenblatt, D. J., and Koch-Weser, J., American Heart fournal, 1973, 86,
478 .
5 Greenblatt, D. J., and Shader, R. I., Current Therapeutic Research, 1972, $14,615$.

6 Epstein, S. A., and Braunwald, E., Annals of Internal Medicine, 1967, 67, 1333.

7 Fitzgerald, J. D., Clinical Pharmacology and Therapeutics, 1969, 10, 292. ${ }^{8}$ Prichard, B. N. C., Lionel, N. O. W., and Richardson, S. A., Postgraduate Medical fournal, 1971, suppl., 47, 59.

\section{Age and the Face}

The rate of decrease in elasticity of the facial skin varies greaily in individuals, some people appearing still quite young at an age when others look positively ancient. Climatic conditions influence the change, and those who have spent years under tropical suns tend to be noticeably lined and creased. Slimming and loss of weight through illness or anxiety will also produce an appearance of accelerated ageing. Not unnaturally, specialist surgical techniques have been developed to try to reverse the process, most times with gratifying results, but sometimes not.

In selection of his patients the wise surgeon avoids those who appear to be psychologically disturbed and those who attribute personal problems to their appearance, whereas he will accept for treatment patients who come to him and plainly state their dislike of their looks and those whose very work depends upon their physical features. General physical health and medical history are also important-patients with a bleeding tendency should be refused such operations.

The patient must be informed of the possible complications of any treatment and the time taken for a full recovery; too often people attend their doctors asking for cosmetic surgery, not knowing anything about the procedures, totally unaware of the risk of complications, and with no idea of the duration of hospital stay needed.

Surgeons tend to discourage patients from having treatment on the N.H.S. and those that are accepted almost always have their names at the end of the waiting list. Regrettably, they tend to stay near the bottom as the more urgent cancer, congenital deformities, and accidents of all kinds are naturally given preference. If the patient insists on early treatment and wants a guarantee that a specific surgeon will operate on her she not infrequently decides to have private treatment.

The operative techniques, though specialized, are fairly standard. The forehead is rarely tackled-only where the furrows are deep and severe. The approach is through an incision on the edge of the hair line, not in the hairline; the skin is difficult to lift off the muscle, and marginal areas of breakdown are not infrequent. A scar can always be seen, though in time it will become visible only to close examination. The so-called "minilift" is only of temporary benefit and is performed in the hair in the upper temporal region on each side. Deep frown marks are removed by direct excision, with undermining of the neighbouring skin to detach the corrugator and procerus muscle attachments. When the frown marks are double an approach is often made in the eyebrow on each side, with undermining of the muscle attachments and division of the nerves. This procedure is helpful, but a permanent paralysis (and therefore permanent cure) cannot be guaranteed, for reinnervation not infrequently occurs.

Upper eyelids can be reduced by excision of the redundant skin wit's a curved incision in the crease of the eyelid. The fatty herniation on the inner side is removed and the sac closed by suture. The lower lids can be reduced by an incision along the margin, then outwards and slightly downwards in one of the "crows feet," and the slack is taken out laterally. 
There are two age groups for lower lid reductions, the young woman with congenital "baggy" eyelids - often noticeable in late adolescence-and the older woman with creased and redundant skin. The lateral scars can be seen for about three months but can easily take cosmetics. There are dangers: corneal abrasion, haematoma in the orbit, and haematoma in the eyelids. Blindness due to spasm or thrombosis of the central artery of the retina has been reported and is probably due to the use of adrenaline with the local anaesthesia which is used extensively outside Britain.

Slack, sagging cheek and slack neck skin can be lifted through an incision in each side which starts in the temporal hairline, passes down immediately in front of the ear, continues up behind the ear on the mastoid skin, and finally curves down in the hairline posteriorly. A new site for the ear must be made in the stretched skin; no tension must be put upon the auricle. The scar is fine and can be seen only on close examination.

Any of these procedures may have complications. A haematoma may occur and may need to be evacuated; rarely selective facial paralysis may occur, which usually recovers spontaneously, and there may be necrosis of skin in isolated areas along the suture lines, but again this is rare. Anaesthesia of the ear and scalp may occur from injury to the great auricular nerve. The scars will take some months to really settle down. Ladies' hairdressers must be taken into confidence, the hair must be dressed down, and chignons are not usually possible. Social and public appearances can usually be made in three or four weeks after operation.

In the elderly one lift will be enough. Middle-aged patients may ask for a repeat in five to six years. The operation is seldom performed before 40 years of age, unless it is for professional purposes, when it may have to be repeated in four to five years' time. The patient who is always smiling and laughing with the really "alive" face is often the most difficult one on whom to obtain a good result, for they form their creases again quite quickly. The dull looking, unemotional face can be lifted with greater lasting effect. A third lift is very difficult, as inelastic fibrous tissue becomes laid down beneath the skin and it is not possible to get as much stretch as required.

In general patients need to be reminded that they are only as young as they feel, and many who search for improvement by plastic surgery vainly expect that treatment will solve a multitude of problems. The Narcissus is seldom completely satisfied; the ugly, creased, but cheerful person is usually very grateful.

\section{Pseudotumours of the Orbit}

The vague term pseudotumour is used to describe a clinical entity with features suggestive of a genuine orbital neoplasm but in which this diagnosis cannot be confirmed. A. BirchHirschfeld, who introduced the term in 1905, included all non-neoplastic disorders; later authors have preferred to confine it to idiopathic inflammatory lesions, while L. E. Zimmerman ${ }^{1}$ suggested that the causes should be divided into lesions of known pathogenesis occuring either as localized orbital swellings or as part of a systemic disorder, together with a third idiopathic group.

Orbital pseudotumours are uncommon as opposed to rare, are usually unilateral, and occur mainly in elderly patients. Some series report a predominance of women. The clinical features mimic those of a tumour, though there is often evidence of inflammation. On some occasions a mass is palpable. Proptosis is common, but not invariable, and seldom exceeds $10 \mathrm{~mm}$; the globe may also be displaced vertically as well as forwards. The vision is usually unaffected but can be diminished. Diplopia is usually present, and indeed may be the last feature to disappear. There is often oedema of the lids and conjunctiva. The onset may be acute or the conditions may progress over several months. Unfortunately none of these features can offer the clinician a clear guide to the diagnosis, and in addition to a full general assessment extensive investigations may be required.

Infections of the paranasal sinuses or a dental abscess may result in orbital cellulitis which terminates in dense scar formation, but this is less common since the introduction of antibiotics. Associated lesions of the respiratory tract and kidney might suggest Wegener's granulomatosis, and disease in other tissues may indicate polyarteritis nodosa, dermatomyositis, Siøgren's syndrome, sarcoidosis, or histiocytosis. Dysthyroid exophthalmos is a condition which some would not class as a pseudotumour, yet it is one of the commonest causes of the characteristic symptoms and signs; many cases present unilaterally making the diagnosis even more difficult.

The orbit is a difficult structure to examine radiologically owing to the surrounding bone-however, in recent years considerable advances have been made. Carotid arteriography combined with subtraction techniques yields "bone free" radiographs which can show the orbital vasculature in great detail. Orbital venography is of great value, and the examination has become much simpler since the introduction of a technique for injecting contrast material into the frontal veins. $^{2}$ In addition there has been a great increase in the use of diagnostic ultrasound, while more recently ultrasound holography ${ }^{3}$ has been introduced, which offers a threedimensional examination.

All the investigations can do, however, is to indicate the presence and position of a space-occupying process, and the next stage in the diagnosis may be an orbital biopsy, particularly if vision is threatened. Histological examination may then confirm lesions occurring as part of a systemic disorder or local disorders such as lipogranuloma, cholesteatoma, dermoid cyst, foreign-body granuloma, fibrous dysplasia of bone, or parasitic infestation such as cysticercosis. The idiopathic group can give problems, especially when lymphoid proliferation is predominant, as there can be a wide spectrum from reactive hyperplasia to malignant lymphoma. The pathological features of all types of pseudotumours were recently well reviewed by A. Garner.4

The prognosis in most cases is good-by definition the patient is spared the consequences of a neoplastic condition. Sometimes specific treatment can be directed towards an associated systemic disease, but more usually treatment with steroids and occasionally antibiotics is tried. As more becomes known about these conditions and specific causes are found the diagnosis of pseudotumour will slowly become rare, but until that time it remains a useful label. The investigation of these patients is both difficult and time-consuming and should be concentrated in those few centres with the necessary facilities.

\footnotetext{
Zimmerman, L. E., Transactions of the American Academy of Ophthalmology and Otolaryngology, 1967, 71, 322.

Vritsios, A., Archives de la Societé d'Ophthalmologie de la Grèce du Nord $1963,12,223$

Wright, J. E., Lloyd, G. A. S., and Clare, A. B., Transactions of Ophthalmological Society of the United Kingdom, 1971, 91, 519.

4 Garner, A., Fournal of Clinical Pathology, 1973, 26, 639.
} 